\title{
Research on comprehensive load vibration test of electric vehicle motor controller unit
}

\author{
Zhibiao Yan ${ }^{1,2,}$, Lingxiao Zhao ${ }^{1,2}$, and Huansheng $\mathrm{Ma}^{1,2}$ \\ ${ }^{1}$ CATARC Automotive Test Center (Tianjin) Co., Ltd, Tianjin 300300, China \\ ${ }^{2}$ China Automotive Technology \& Research Center Co., Ltd, Tianjin 300300, China
}

\begin{abstract}
In this article, the vibration test standards and general test methods for electric vehicle motor control unit are introduced. New vibration test conditions are proposed based on the vibration data collected during the actual vehicle test. Using the vehicle output current data as the electrical load of the motor controller unit, and the temperature cycle as the environmental load, a comprehensive load vibration test method that integrates the electrical load and the environmental load is proposed. According to the test method, the equipment and test process of the comprehensive on-load vibration test of environmental load are determined. Summarized three important principles in the design of fixtures: lightweight, low damping, and no resonance. A novel testing method for motor controllers is proposed.
\end{abstract}

Keywords: Electric car; Motor controller unit; Test method.

\section{Preface}

In the 21 st century, with the development of China's auto industry and the increase in the scale of China's auto market, environmental pollution caused by the large-scale use of traditional cars has become a serious social problem. Problems such as declining air quality and depletion of petroleum energy have begun to affect China's economic and social development [1]. Therefore, in 2001, China compiled electric vehicle technology into an important research direction during the "Tenth Five-Year Plan" period. By 2020, China's electric vehicle sales have increased to 850,000 , and the growth rate is rapid. Electric vehicles have become the most important part of China's automobile industry[2]. At the same time, a large number of automobile consumers in China have put forward more stringent requirements on the durability, safety, and stability of electric vehicles during use.

Electric vehicle motor controller unit is a complex electrical system composed of a variety of electronic components. As a component that controls the power output of an electric vehicle, the motor controller unit plays a decisive role in the overall performance. On the one hand, as an important part of electric vehicles, the motor controller unit must be reliable; on the other hand, the motor controller unit has a complex structure and numerous electronic components. In recent years, the motor controller unit and other components such as the motor are often highly integrated. The harsh working environment and complex

* Corresponding author: yanzhibiao@catarc.ac.cn 
structure of the motor controller unit have caused great challenges to its reliability and the overall safety of electric vehicles.

At present, when verifying the reliability of the motor controller unit through simulation, it is necessary to use the method of multi-physics coupling analysis. In the simulation, it is necessary to consider the influence of electric stress, self-heating, environmental temperature and humidity conditions, and vibration conditions. This is very difficult to establish an accurate model and analyze. In view of this, the comprehensive load test of the motor controller unit is an important method in the reliability design and inspection of the motor controller unit.

\section{Related test standards and methods}

China's national test standard for electric vehicle motor control is GB/T18488. The current version is the 2015 edition: GB/T 18488.1-2015 "Drive motor system for electric vehicles Part 1:Test methods" and GB/T 18488.2-2015 "Drive motor system for electric vehicles Part 2:Test methods" [3-4].

\subsection{Vibration test}

Vibration test simulates the performance of samples under the influence of external vibration in real working conditions, which is a crucial test method in the reliability research of auto parts.

For the motor controller unit, the vibration load mainly comes from the unsteady vibration of the road passing through the suspension system and the steady vibration of rotating parts such as the motor and transmission. Unsteady vibration is affected by the road surface, and the vibration excitation is randomly distributed in the time domain and frequency. Therefore, broadband random vibration is used to simulate unsteady vibration in the vibration test; The frequency of steady state vibration is related to the motor speed. The vibration peak value changes with the speed in the frequency domain to show an obvious order relationship, showing regularity. In the vibration test, the sinusoidal frequency sweep vibration is used to simulate [5].Applying different vibration loads to the sample can test the reliability of the sample in different actual use situations.

\subsubsection{Sinusoidal vibration test}

The 2006 edition of GB/T18488 quoted the Chinese automotive industry standard QC/T 413-2002 Basic Technical Conditions of Automotive Electrical Equipment for the vibration test of motor controller units. Compared with QC/T 413-1999, which was replaced by QC/T 413-2002, the "fixed frequency vibration" is deleted in the sinusoidal vibration section but the "sweep frequency vibration" content is added. Intensified attention has been paid to the influence of order wide-frequency band vibration excitation generated by the variable-speed motion of rotating parts in automobiles [6].

The 2015 edition of GB/T18488 quoted the Chinese national test standard GB/T2423.10 -2008 "Environmental testing for electric and electronic products Part 2: Tests methods Test Fc: Vibration (sinusoidal)" as the sinusoidal vibration test method.

The severity of the standard test for sinusoidal vibration is controlled by parameters such as frequency, vibration acceleration amplitude, frequency sweep speed, and test time. The conditions are shown in Table 1. 
Table 1. Sinusoidal vibration test conditions.

\begin{tabular}{|c|c|c|c|c|c|}
\hline $\begin{array}{l}\text { Installation } \\
\text { location }\end{array}$ & $\begin{array}{c}\text { Frequency } \\
\quad / \mathrm{Hz}\end{array}$ & $\begin{array}{c}\text { Vibration } \\
\text { amplitude } / \mathrm{mm}\end{array}$ & $\begin{array}{c}\text { Vibration } \\
\text { acceleration } \\
\text { amplitude } /\left(\mathrm{m} / \mathrm{s}^{2}\right)\end{array}$ & $\begin{array}{r}\text { Frequency } \\
\text { sweep speed } \\
/(\text { otc } / \text { min })\end{array}$ & $\begin{array}{l}\text { Test } \\
\text { time } / \mathrm{h}\end{array}$ \\
\hline \multirow{2}{*}{ Engine } & $10-50$ & 2.5 & \multirow{2}{*}{--} & \multirow{2}{*}{8} & \multirow{2}{*}{8} \\
\hline & $50-200$ & 0.16 & & & \\
\hline \multirow{2}{*}{ Other location } & $10-25$ & 1.2 & -- & \multirow{2}{*}{8} & \multirow{2}{*}{8} \\
\hline & $25-500$ & -- & 30 & & \\
\hline
\end{tabular}

In the electric vehicle motor controller unit test, there is no engine in the electric vehicle structure, and the test conditions generally quote "other location".

\subsubsection{Random vibration test}

Compared with the previous version of 2006, GB/T18488-2015 adds random vibration content and pays more attention to the impact of unsteady excitation. The random vibration test method refers to the national standard GB/T 28046.3-2011 "Road vehiclesEnvironmental conditions and testing for electrical and electronic equipment-Part 3: Mechanical loads", according to the installation location, the test conditions related to electric vehicle motor controller units are cited among them "Test IV-Passenger car elastomer (body)" [7].

\subsection{Environmental test}

The environmental test simulates the reliability of the tested sample under extreme weather conditions. GB/T18488-2015 "Drive Motor System for Electric Vehicles" divides the environmental test into three parts: low temperature reliability test, high temperature reliability test, and damp heat reliability test.

Winters in northern China are long and extreme cold weather occurs frequently. In order to verify the impact on the reliability of electric vehicles under cold weather conditions, the low temperature reliability test requires the test at $-40^{\circ} \mathrm{C}$. In southern China, the summer is hot, with persistent high temperatures often occurring. At the same time, some provinces along the southeast coast have humid climate and frequent rainfall. The working environment of auto parts is often harsher. In order to verify the adverse effects of high temperature and high humidity on auto parts, the high temperature reliability test requires the ambient temperature to be above $85^{\circ} \mathrm{C}$ for testing, and the humid heat test requires $40^{\circ} \mathrm{C}$ relative humidity $90 \% \sim 95 \%$ conditions to verify reliability.

\section{Comprehensive load vibration test conditions}

\subsection{Vibration test conditions}

\subsubsection{Sinusoidal vibration test conditions}

The function of the sine frequency sweep test is to check the reliability of the sample subjected to vibration excitation of a stable frequency. The steady-state frequency vibration load received by the motor controller unit mainly comes from the vibration generated by 
the motor during rotation, and the vibration generated by the motor is more severe when accelerating.

When a real vehicle accelerates on a flat road, a vibration acceleration sensor is arranged on the motor to collect its vibration signal. After the vibration signal is Fourier transformed, the relationship between the vibration acceleration in the $\mathrm{X}, \mathrm{Y}$, and $\mathrm{Z}$ directions of the motor and the speed and frequency is shown in Figure 1.
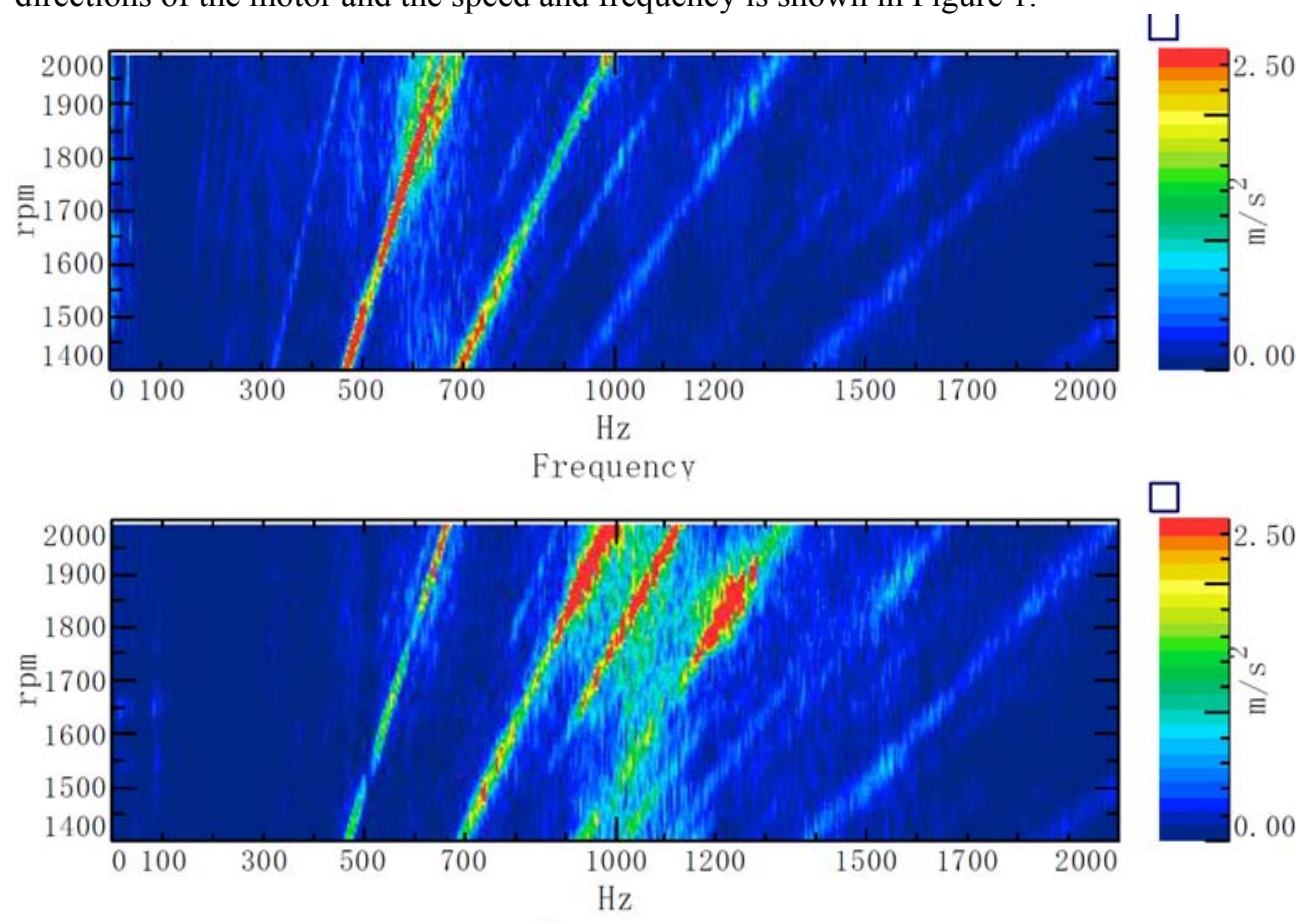

Frequency

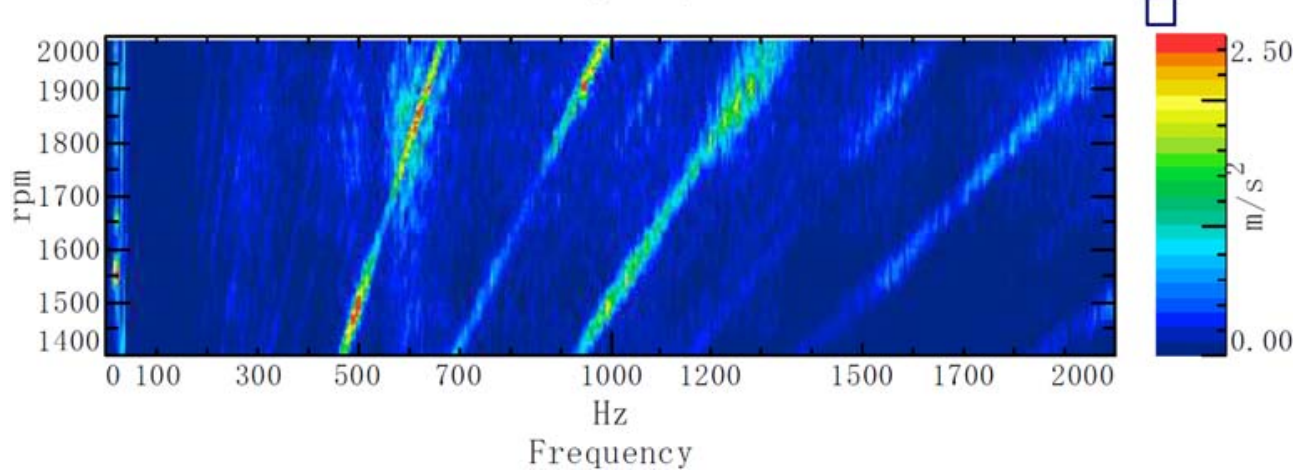

Fig. 1. Motor vibration diagram under real vehicle acceleration conditions.

The peak value of motor vibration is below $1500 \mathrm{~Hz}$. In the sinusoidal test, the upper frequency limit is set to $1500 \mathrm{~Hz}$.

\subsubsection{Random vibration test conditions}

The random vibration test method quoted the Chinese national standard GB/T 28046.32011 "Road vehicles-Environmental conditions and testing for electrical and electronic equipment-Part 3: Mechanical loads" "Test IV-Passenger Car Elastomers (Body)" 
Provisions. The standard requires 8 hours of vibration in each direction, the RMS value of vibration acceleration is $27.68 \mathrm{~m} / \mathrm{s}^{2}$, and the relationship between power spectral density and frequency is shown in Table 2.

Table 2. Power spectral density and frequency relation table.

\begin{tabular}{|c|c|}
\hline Frequency/Hz & $\mathbf{P S D}\left(\mathbf{m} / \mathbf{s}^{\mathbf{2}}\right)^{\mathbf{2}} \mathbf{H z}$ \\
\hline 10 & 20 \\
\hline 50 & 6.5 \\
\hline 180 & 0.25 \\
\hline 300 & 0.25 \\
\hline 360 & 0.14 \\
\hline 1000 & 0.14 \\
\hline
\end{tabular}

\subsection{Environmental test conditions}

The structure of the motor controller unit is complex. The shell is mostly made of metal materials such as steel and aluminum. There are copper conductive parts inside, as well as many electronic components such as PCB board and many inductors, capacitors, chips and so on soldered on the PCB board. Various components have complex structures and different materials, and their thermal conductivity, material strength, and thermal expansion coefficient are different, showing different thermal stress states.

In the environmental test, a single high temperature or low temperature reliability test can no longer meet the increasingly stringent reliability requirements, so GB/T 28046.42011 "Road vehicles-Environmental conditions and testing for electrical and electronic equipment-Part 4: Climatic loads" 5.3.1 The temperature cycle of the specified rate of change is used as the environmental condition of the comprehensive load vibration test. The test conditions are shown in Figure 2 [8].

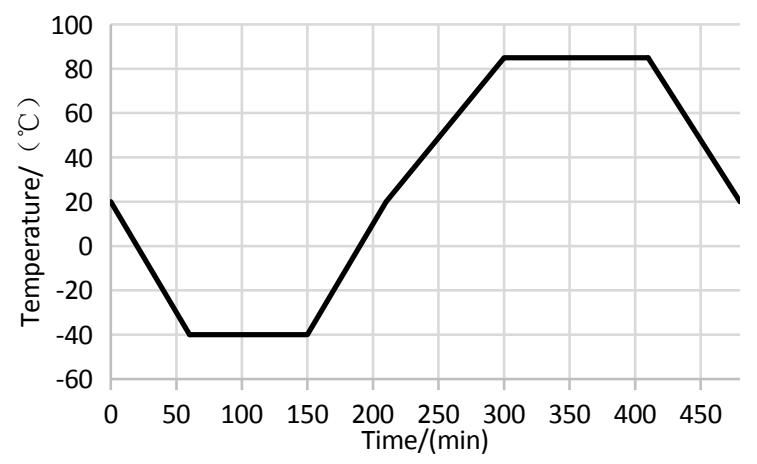

Fig. 2. Environmental test conditions.

In the comprehensive load vibration test, due to the harsh environmental test conditions, the vibration sensor and its wiring harness must be able to withstand the extreme high and low temperatures in the environmental conditions. Otherwise, the feedback signal of the vibration test will be inaccurate and the test will fail.

\subsection{Load condition}

In the process of real vehicle driving, due to the complex road conditions, electric vehicles often cannot drive at a constant speed at the rated speed, especially in urban road conditions 
where the road is congested and the car starts and stops frequently. The working conditions of the motor controller unit are also more complicated, and the electrical stresses on the internal electronic components are also more complicated. The continuous output of the rated current for a long time during the test obviously cannot fit the actual load environment, and it is difficult to meet the test requirements.

In order to simulate the real driving situation, by monitoring the current of the motor controller unit in the actual vehicle test, the output current data of driving under different road conditions can be summarized, and a representative vehicle controller output working condition can be obtained, as shown in the figure 3 .

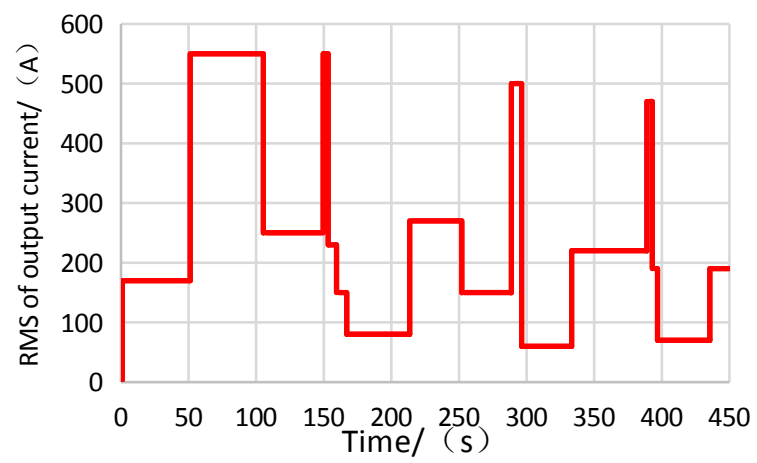

Fig. 3. Motor controller unit output working condition curve.

\section{Comprehensive load vibration test process}

\subsection{Test subject process}

There are strict requirements for the control and test equipment and related parts of the integrated load vibration test process. The main body is divided into the production of fixtures, the inspection of sample before test, the layout of the loading system, the installation of the sample fixture, the layout of the chamber, the connection of the loading system, the activation of the chamber parameter setting, the setting and starting of the vibration bench parameter, and the parameter setting of the electrical test equipment. Change the vibration direction, complete the test, arrange and dismantle the equipment, etc. The actual installation diagram of the test sample is shown in Figure 4, and the specific flowchart is shown in Figure 5.

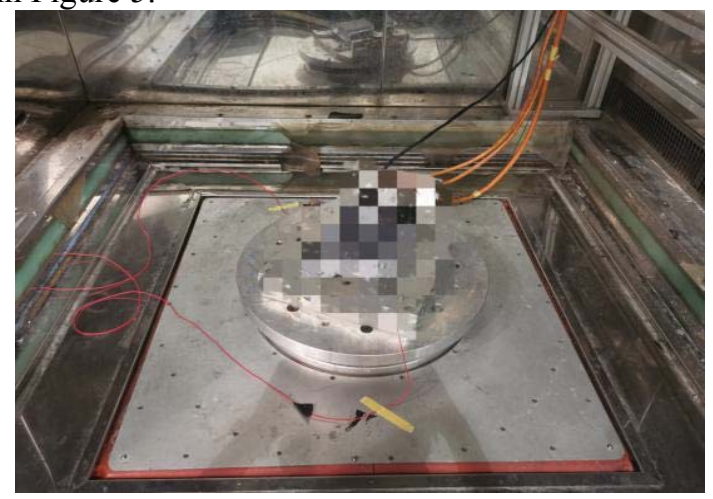

Fig. 4. Actual installation of test samples. 


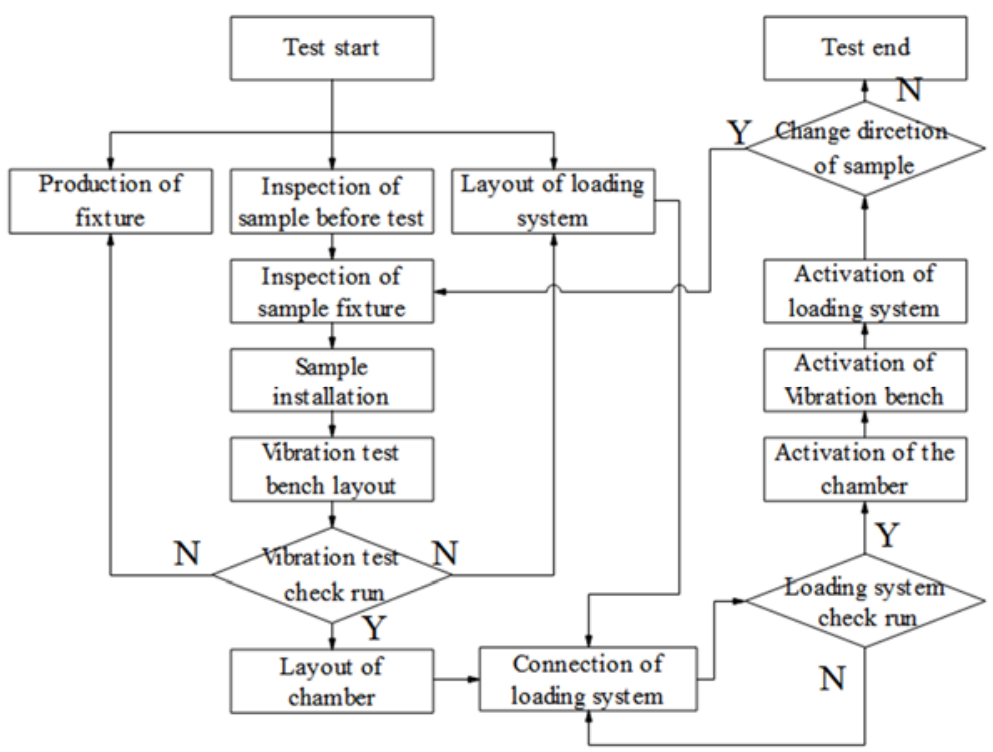

Fig. 5. Flow chart of test subject.

\subsection{Electrical load test system}

According to the parameters of the motor controller unit, select electrical equipment to build a test system to simulate the working environment of the motor controller unit on the entire vehicle. The programmable high-voltage DC power supply simulates the battery pack on the real car; The inductive load simulates the motor on the vehicle, The low-voltage power supply supplies power to the control circuit of the motor controller unit, The chiller is connected to the controller for heat dissipation. The test control system with CAN communication module uses CAN bus technology to communicate with the motor controller unit, simulating VCU sending output current, frequency and other commands to the motor controller unit to control the output working condition. The schematic diagram of electrical load test systemare shown in Figure 6.

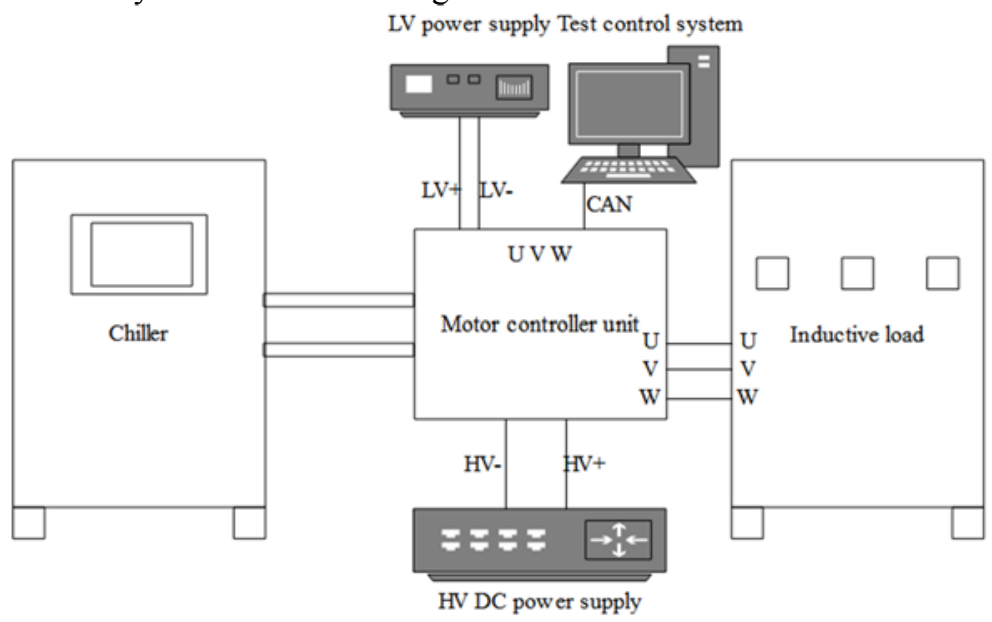

Fig. 6. Electrical load test system. 
If the test needs to record power supply voltage, chiller flow temperature and other parameters, also need to use equipment with remote communication capabilities. By integrating various devices into the test system for common control, the degree of automation of the test is improved.

\section{Three key points in fixture design}

The fixture is the link between the vibration test bench and the test sample. The performance of the fixture will directly affect the test effect of the vibration test. The following three issues should be paid attention to in the design of fixtures.

\subsection{Lightweight}

In the vibration test, the fixture as an important auxiliary part of the fixed sample and the vibration test bench will also participate in the vibration of the test. According to Newton's second law, the thrust required for vibration test is shown in formula (1).

$$
F=\left(m_{0}+m_{1}+m_{2}+m_{3}\right) a
$$

Among them: $F$ is the thrust required for the test; $m_{0}$ is the mass of the vibration bench moving coil; $m_{1}$ is the quality of the vibration bench table; $m_{2}$ is the quality of the fixture; $m_{3}$ the mass of the vibration test sample; $a$ is the vibration acceleration

In the case of the vibration test bench unchanged, when the moving coil mass and table quality of the vibration test bench are constant, the greater the quality of the fixtures, the smaller the sample mass that the vibration test bench can withstand. Therefore, when the mass of the sample is large or when multiple samples are tested at the same time, the lightweight design of the fixture should be paid attention to to meet the test requirements. The lightweight optimization design of a vibration fixture with excessive mass is carried out, and the design results obtained are shown in Figure 7.

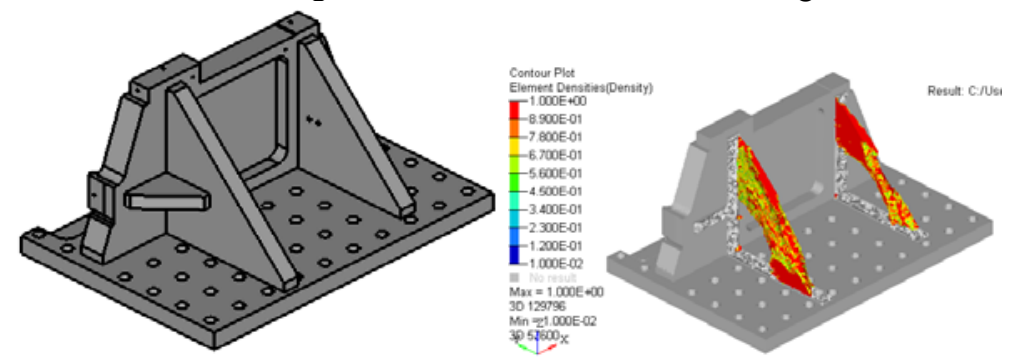

Fig. 7. Fixture before lightweight optimization and optimization results.

\subsection{Low damping}

IThe fixture plays the role of connecting the sample and the vibration test bench in the test. If the fixture is regarded as a single degree of freedom vibration system of spring and damping during the vibration test, the movement of the fixture is shown in formula (2).

$$
m \ddot{X}+C \dot{X}+K X=F(t)
$$


Among them: $m$ is the mass; $C$ is the viscous damping coefficient; $K$ is the stiffness; $F(t)$ is the external force; $X$ is the displacement; $\dot{X}$ is the first derivative of displacement with respect to time; $\ddot{X}$ is the second derivative of displacement with respect to time.

When the system is excited by a steady-state sinusoid, the amplitude A of the system vibration is shown in formula (3):

$$
A=\frac{1}{\sqrt{\left(1-\bar{\omega}^{2}\right)^{2}+(2 \xi \bar{\omega})^{2}}} \cdot \frac{F_{v}}{K}
$$

Among them: $\bar{\omega}=\omega / \omega_{n} ; \omega_{\mathrm{n}}^{2}=k / m ; C / 2 \mathrm{~m}=\xi \omega_{\mathrm{n}} ; F_{\mathrm{v}} / K$ is the static displacement under $F_{\mathrm{v}}$.

It can be seen that the damping coefficient $\xi$ has a very large influence on the vibration system amplitude. If the damping of the fixture is too large, the transmitted vibration intensity will be weakened, which will affect the test effect.

\subsection{No resonance}

Mode is an inherent dynamic characteristic of the structure, and the vibration characteristics exhibited at different frequencies are called mode shapes. Each modal frequency corresponds to a unique mode shape. When excited by this modal frequency, the structure will vibrate violently according to the mode shape, which is called "resonance."

According to the theory of mechanical vibration, for a free vibration system with $\mathrm{N}$ degrees of freedom, the differential equation of the system is shown in equation (4)

$$
M\{\ddot{x}\}+C\{\dot{x}\}+K\{x\}=0
$$

Among them: $M$ is the mass matrix; $K$ is the stiffness matrix; $C$ is the damping matrix; $\{\ddot{x}\}$ is the acceleration column vector; $\{\dot{x}\}$ is the velocity column vector; $\{x\}$ is the displacement column vector.

For structures with low damping, $C$ is usually ignored. Therefore, the differential equation of a vibration system that ignores the effect of damping is shown in equation (5).

$$
M\{\ddot{x}\}+K\{x\}=0
$$

According to the characteristics of the second-order linear homogeneous differential equation, assuming $x_{i}=q_{i} \sin \omega_{i} t$, substituting formula (5) into (6) can be obtained.

$$
\left(-\omega_{i}^{2} M+K\right)\left\{q_{i}\right\}=0
$$

If it has a non-zero solution, as shown in equation (7).

$$
\left|-\omega_{i}^{2} M+K\right|=0
$$


This equation is called the characteristic equation of the mechanical vibration system, which is the $\mathrm{N}$-th order equation of $\omega_{i}^{2}$. The N-order characteristic equation of the vibration system corresponds to $\mathrm{N}$ different non-zero solutions, that is, the modal frequency of the system. If the fixture is here When the structure is excited, resonance will appear [9].

In the fixture design stage, modal simulation and optimization should be carried out to determine that its natural frequency distribution is far away from the vibration test frequency to save trial costs and ensure test quality.

\section{Conclusion}

The motor controller unit is affected by the electrical load, mechanical load, and climate load when it is working, and the working conditions are harsh. However, as a key component of an electric vehicle, it needs to have good reliability. This article summarizes the three main loads of motor controllers. These three loads are vibration mechanical load, temperature cycle climate load and electrical load close to actual working conditions. According to three kinds of loads, a reliability test method is proposed. Summarize the test system composition required in the key test process test according to the method. And deduced the important light weight, low damping, and no resonance principles in the vibration mechanical load test. A new idea is proposed for the reliability test of the motor controller.

\section{References}

1. Energy-saving and new energy automobile industry development plan [J] China Motor, 2012(08):60-67.

2. ZHAO Y F, YANG W S, LI R J, SONG X M. Analysis on Development Trend of Pure Electric Vehicles in China[J]. Auto Engineer, 2020(07):14-17.

3. GB/T 18488.1-2015 Drive motor system for electric vehicles Part 1:Test methods[S].Beijing: Standards Press of China,2015

4. GB/T 18488.2-2015 Drive motor system for electric vehicles Part 2:Test methods[S].Beijing: Standards Press of China,2015

5. HUANG X, HE P L, CAI Z T. Research on Evaluation Methods of Random Vibration of EV Drive Motor System[J] . Auto Electric Parts,2018(01):10-13+15.

6. GAO S, GAO Q.Study on Vibration Test Method for Electrical Machine and controllers of Electric Vehicles[J]. Environmental Technology,2013,31(01):24-28.

7. GB/T 28046.3-2011 Road vehicles-Environmental conditions and testing for electrical and electronic equipment-Part 3: Mechanical loads [S].Beijing: Standards Press of China,2011

8. GB/T 28046.4-2011 Road vehicles-Environmental conditions and testing for electrical and electronic equipment-Part 4:Climatic loads [S].Beijing: Standards Press of China,2011.

9. SU S J, XU Y L, ZHANG Z Y. Modal analysis of automobile wheels based on different materials[J]. Automobile Applied Technology,2018(19):225-226+235. 